\title{
Reliability assessment of generating systems with wind power penetration via BPSO
}

\begin{abstract}
The growing pervasiveness of the Wind Energy Conversion System (WECS) in power systems has a great influence on the electrical system reliability in relation to other conventional sources for power generation. This current study offers a binary particle swarm optimisation (BPSO) application with Weibull model to reliably evaluate the generation systems with a WECS. The proposed methodology is based on hourly time series wind speed and uses Weibull model and simulation of the operation of generation system, taking into consideration the random failures of conventional units of the system and the fluctuating wind energy of a WECS. The BPSO algorithm adopts intelligent research to explore the meaningful system states and accelerate their integrated convergence, so that makes it feasible to locate all possible failure states in the system states space in order to calculate the reliability indices with WECS. The numerical simulation of the suggested solution is compared with the established Monte Carlo simulation (MCS). The reliability test system (IEEE-RTS-79) is employed to show the effectiveness of the proposed algorithm.
\end{abstract}

Keyword: Reliability assessment; Wind power; Generating system; Binary particle swarm optimisation; Weibull model 